This is the post print version of the article, which has been published in Information Processing Letters . 2018, 129 (1), 11-15. http://dx.doi.org/ 10.1016/j.ipl.2017.08.006.

[Th This document has been downloaded from TamPubuta.fi

\title{
A hardness result and new algorithm for the longest common palindromic subsequence problem
}

\author{
Shunsuke Inenaga \\ Department of Informatics, Kyushu University, Japan \\ Heikki Hyyrö \\ School of Information Sciences, University of Tampere, Finland
}

\begin{abstract}
The 2-LCPS problem, first introduced by Chowdhury et al. [Fundam. Inform., 129(4):329-340, 2014], asks one to compute (the length of) a longest palindromic common subsequence between two given strings $A$ and $B$. We show that the 2-LCPS problem is at least as hard as the well-studied longest common subsequence problem for 4 strings. Then, we present a new algorithm which solves the 2-LCPS problem in $O\left(\sigma M^{2}+n\right)$ time, where $n$ denotes the length of $A$ and $B, M$ denotes the number of matching positions between $A$ and $B$, and $\sigma$ denotes the number of distinct characters occurring in both $A$ and $B$. Our new algorithm is faster than Chowdhury et al.'s sparse algorithm when $\sigma=o\left(\log ^{2} n \log \log n\right)$.
\end{abstract}

Keywords: palindromes, longest common subsequences, nesting rectangles

\section{Introduction}

Given $k \geq 2$ string, the longest common subsequence problem for $k$ strings ( $k$-LCS problem for short) asks to compute (the length of) a longest string that appears as a subsequence in all the $k$ strings. Whilst the problem is known to be NP-hard for arbitrary many strings [1], it can be solved in polynomial time for a constant number of strings (namely, when $k$ is constant).

The 2-LCS problem that concerns two strings is the most basic, but also the most widely studied and used, form of longest common subsequence computation. Indeed, the 2-LCS problem and similar two-string variants are central topics in theoretical computer science and have applications e.g. in computational biology, spelling correction, optical character recognition and file versioning. The fundamental solution to the 2-LCS problem is based on dynamic programming [2] and takes $O\left(n^{2}\right)$ for two given strings of length $n^{1}$. Using the so-called

Email addresses: inenaga@inf.kyushu-u.ac.jp (Shunsuke Inenaga), heikki.hyyro@uta.fi (Heikki Hyyrö)

${ }^{1}$ For simplicity, we assume that input strings are of equal length $n$. However, all algorithms mentioned and proposed in this paper are applicable for strings of different lengths. 
"Four Russians" technique [3], one can solve the 2-LCS problem for strings over a constant alphabet in $O\left(n^{2} / \log ^{2} n\right)$ time [4]. For a non-constant alphabet, the 2-LCS problem can be solved in $O\left(n^{2} \log \log n / \log ^{2} n\right)$ time [5]. Despite much effort, these have remained as the best known algorithms to the 2-LCS problem, and no strongly sub-quadratic time 2-LCS algorithm is known. Moreover, the following conditional lower bound for the 2-LCS problem has been shown: For any constant $\lambda>0$, an $O\left(n^{2-\lambda}\right)$-time algorithm which solves the 2-LCS problem over an alphabet of size 7 refutes the so-called strong exponential time hypothesis (SETH) [6].

In many applications it is reasonable to incorporate additional constraints to the LCS problem (see e.g. [7, 8, 9, 10, 11, 12, 13, 14, 15, 16]). Along this line of research, Chowdhury et al. [17] introduced the longest common palindromic subsequence problem for two strings (2-LCPS problem for short), which asks one to compute (the length of) a longest common subsequence between strings $A$ and $B$ with the additional constraint that the subsequence must be a palindrome. The problem is equivalent to finding (the length of) a longest palindrome that appears as a subsequence in both strings $A$ and $B$. Chowdhury et al. presented two algorithms for solving the 2-LCPS problem. The first is a conventional dynamic programming algorithm that runs in $O\left(n^{4}\right)$ time and space. The second uses sparse dynamic programming and runs in $O\left(M^{2} \log ^{2} n \log \log n+n\right)$ time and $O\left(M^{2}\right)$ space $^{2}$, where $M$ is the number of matching position pairs between $A$ and $B$.

The contribution of this paper is two-folds: Firstly, we show a tight connection between the 2-LCPS problem and the 4-LCS problem by giving a simple linear-time reduction from the 4-LCS problem to the 2-LCPS problem. This means that the 2-LCPS problem is at least as hard as the 4-LCS problem, and thus achieving a significant improvement on the 2-LCPS problem implies a breakthrough on the well-studied 4-LCS problem, to which all existing solutions $[18,19,20,21,22]$ require at least $O\left(n^{4}\right)$ time in the worst case. Secondly, we propose a new algorithm for the 2-LCPS problem which runs in $O\left(\sigma M^{2}+n\right)$ time and uses $O\left(M^{2}+n\right)$ space, where $\sigma$ denotes the number of distinct characters occurring in both $A$ and $B$. We remark that our new algorithm is faster than Chowdhury et al.'s sparse algorithm [17] when $\sigma=o\left(\log ^{2} n \log \log n\right)$.

\section{Preliminaries}

\subsection{Strings}

Let $\Sigma$ be an alphabet. An element of $\Sigma$ is called a character and that of $\Sigma^{*}$ is called a string. For any string $A=a_{1} a_{2} \cdots a_{n}$ of length $n,|A|$ denotes its length, that is, $|A|=n$.

\footnotetext{
${ }^{2}$ The original time bound claimed in [17] is $O\left(M^{2} \log ^{2} n \log \log n\right)$, since they assume that the matching position pairs are already computed. For given strings $A$ and $B$ of length $n$ each over an integer alphabet of polynomial size in $n$, we can compute all matching position pairs of $A$ and $B$ in $O(M+n)$ time.
} 
For any string $A=a_{1} \cdots a_{m}$, let $A^{R}$ denote the reverse string of $A$, namely, $A^{R}=a_{m} \cdots a_{1}$. A string $P$ is said to be a palindrome iff $P$ reads the same forward and backward, namely, $P=P^{R}$.

A string $S$ is said to be a subsequence of another string $A$ iff there exist increasing positions $1 \leq i_{1}<\cdots<i_{|S|} \leq|A|$ in $A$ such that $S=a_{i_{1}} \cdots a_{i_{|S|}}$. In other words, $S$ is a subsequence of $A$ iff $S$ can be obtained by removing zero or more characters from $A$.

A string $S$ is said to be a common subsequence of $k$ strings $(k \geq 2)$ iff $S$ is a subsequence of all the $k$ strings. $S$ is said to be a longest common subsequence (LCS) of the $k$ strings iff other common subsequences of the $k$ strings are not longer than $S$. The problem of computing (the length of) an LCS of $k$ strings is called the $k$-LCS problem.

A string $P$ is said to be a common palindromic subsequence of $k$ strings $(k \geq 2)$ iff $P$ is a palindrome and is a subsequence of all these $k$ strings. $P$ is said to be a longest common palindromic subsequence ( $L C P S$ ) of the $k$ strings iff other common palindromic subsequences of the $k$ strings are not longer than $P$.

In this paper, we consider the following problem:

Problem 1 (The 2-LCPS problem) Given two strings $A$ and $B$, compute (the length of) an LCPS of $A$ and $B$.

For two strings $A=a_{1} \cdots a_{n}$ and $B=b_{1} \cdots b_{n}$, an ordered pair $(i, j)$ with $1 \leq i, j \leq n$ is said to be a matching position pair between $A$ and $B$ iff $a_{i}=b_{j}$. Let $M$ be the number of matching position pairs between $A$ and $B$. We can compute all the matching position pairs in $O(n+M)$ time for strings $A$ and $B$ over integer alphabets of polynomial size in $n$.

\section{Reduction from 4-LCS to 2-LCPS}

In this section, we show that the 2-LCPS problem is at least as hard as the 4-LCS problem.

Theorem 1 The 4-LCS problem can be reduced to the 2-LCPS problem in linear time.

Proof. Let $A, B, C$, and $D$ be 4 input strings for the 4-LCS problem. We wish to compute an LCS of all these 4 strings. For simplicity, assume $|A|=$ $|B|=|C|=|D|=n$. We construct two strings $X=A^{R} Z B$ and $Y=C^{R} Z D$ of length $4 n+1$ each, where $Z=\$^{2 n+1}$ and $\$$ is a single character which does not appear in $A, B, C$, or $D$. Then, since $Z$ is a common palindromic subsequence of $X$ and $Y$, and since $|Z|=2 n+1$ while $|A|+|B|=|C|+|D|=2 n$, any LCPS of $X$ and $Y$ must be at least $2 n+1$ long containing $Z$ as a substring. This implies that the alignment for any LCPS of $X$ and $Y$ is enforced so that the two $Z$ 's in $X$ and $Y$ are fully aligned. Since any LCPS of $X$ and $Y$ is a palindrome, it must be of form $T^{R} Z T$, where $T$ is an LCS of $A, B, C$, and $D$. Thus, we can solve the 4 -LCS problem by solving the 2-LCPS problem. 
Example 1 Consider 4 strings $A=$ aabbccc, $B=$ aabbcaa, $C=$ aaabccc, and $D=$ abcbbbb of length 7 each. Then, an LCPS of $X=$ cccbbaa $\$ 15$ aabbcaa and $Y=$ cccbaaa $\$^{15}$ abcbbbb is cba $\$^{15}$ abc, which is obtained by e.g., the following alignment:

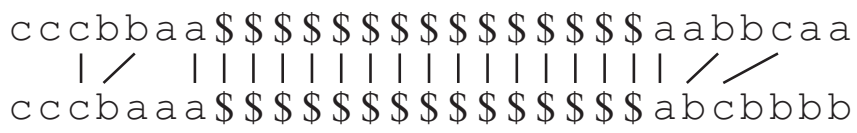

Observe that abc is an LCS of $A, B, C$, and $D$.

\section{A new algorithm for 2-LCPS}

In this section, we present a new algorithm for the 2-LCPS problem.

\subsection{Finding rectangles with maximum nesting depth}

Our algorithm follows the approach used in the sparse dynamic programming algorithm by Chowdhury et al. [17]: They showed that the 2-LCPS problem can be reduced to a geometry problem called the maximum depth nesting rectangle structures problem ( $M D N R S$ problem for short), defined as follows:

\section{Problem 2 (The MDNRS problem)}

Input: A set of integer points $(i, k)$ on a $2 \mathrm{D}$ grid, where each point is associated with a color $c \in \Sigma$. The color of a point $(i, k)$ is denoted by $c_{i, k}$.

Output: A largest sorted list $L$ of pairs of points, such that

1. For any $\langle(i, k),(j, \ell)\rangle \in L, c_{i, j}=c_{j, \ell}$, and

2. For any two adjacent elements $\langle(i, k),(j, \ell)\rangle$ and $\left\langle\left(i^{\prime}, k^{\prime}\right),\left(j^{\prime}, \ell^{\prime}\right)\right.$ in $L, i^{\prime}>i$, $k^{\prime}>k, j^{\prime}<j$, and $\ell^{\prime}<\ell$.

Consider two points $(i, k),(j, \ell)$ in the grid such that $i<j$ and $k<\ell$ (see also Figure 1). Imagine a rectangle defined by taking $(i, k)$ as its lower-left corner and $(j, \ell)$ as its upper-right corner. Clearly, this rectangle can be identified as the pair $\langle(i, k),(j, \ell)\rangle$ of points. Now, suppose that $i$ and $k$ are positions of one input string $A=a_{1} \cdots a_{m}$ and $j$ and $\ell$ are positions of the other input string $B=b_{1} \cdots b_{n}$ for the 2-LCPS problem. Then, the first condition $c_{i, j}=c_{j, \ell}$ for any element in $L$ implies that $a_{i}=a_{k}=b_{k}=b_{\ell}$, namely, $i, j, k, \ell$ are matching positions in $A$ and $B$. Meanwhile, the second condition $i^{\prime}>i, k^{\prime}>k, j^{\prime}<j$, and $\ell^{\prime}<\ell$ implies that $i^{\prime}, j^{\prime}, k^{\prime}, \ell^{\prime}$ are matching positions that are "inside" $i, j, k, \ell$. Hence if we define the set of $2 \mathrm{D}$ points $(i, k)$ to consist of the set of matching position pairs between $A$ and $B$ and then solve the MDNRS problem, the solution list $L$ describes a set of rectangles with maximum nesting depth, and the characters that correspond to the lower-left and upper-right corner matching position pairs define an LCPS netween the input strings $A$ and $B$. 
Recall that $M$ is the number of such pairs. As here the lower-left and upperright corners of each rectangle correspong to matching position pairs, the overall number of unique rectangles in this type of MDNRS problem is $O\left(M^{2}\right)$.

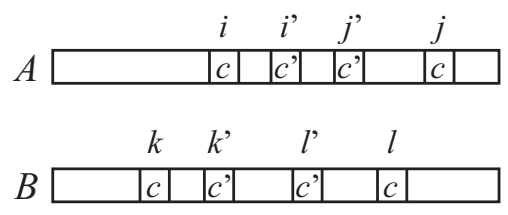

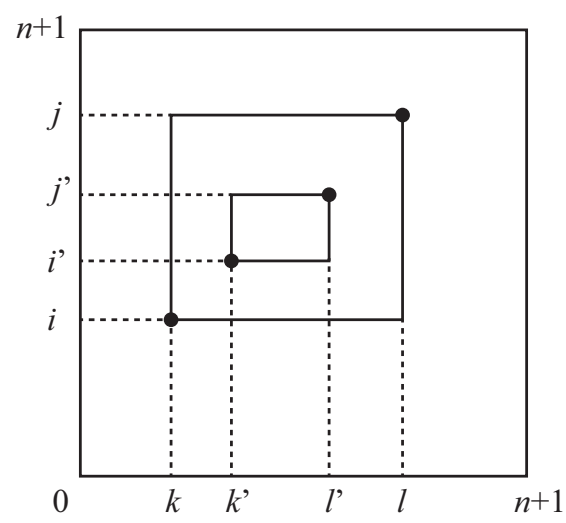

Figure 1: Illustration for the relationship between the 2-LCPS problem and the MDNRR problem. The two nesting rectangles defined by $\langle(i, k),(j, \ell)\rangle$ and $\left\langle\left(i^{\prime}, k^{\prime}\right),\left(j^{\prime}, \ell^{\prime}\right)\right\rangle$ correspond to a common palindromic subsequence $c c^{\prime} c^{\prime} c$ of $A$ and $B$, where $c=c_{i, k}=c_{j, \ell}$ and $c^{\prime}=$ $c_{i^{\prime}, k^{\prime}}=c_{j^{\prime}, \ell^{\prime}}$.

\subsection{Our new algorithm}

Consider the MDNRS over the set of $2 \mathrm{D}$ points $(i, k)$ defined by the matching position pairs between $A$ and $B$, as described above.

The basic strategy of our algorithm is to process from larger rectangles to smaller ones. Given a rectangle $R=\langle(i, k),(j, \ell)\rangle$, we locate for each character $c \in \Sigma$ a maximal sub-rectangle $\left\langle\left(i^{\prime}, k^{\prime}\right),\left(j^{\prime}, \ell^{\prime}\right)\right\rangle$ in $R$ that is associated to character $c$ (namely, $c_{i^{\prime}, k^{\prime}}=c_{j^{\prime}, \ell^{\prime}}=c$ ). The following lemma is important:

Lemma 1 For any character $c \in \Sigma$, its maximal sub-rectangle is unique (if it exists).

Proof. Assume on the contrary that there are two distinct maximal subrectangles $\left\langle\left(i^{\prime}, k^{\prime}\right),\left(j^{\prime}, \ell^{\prime}\right)\right\rangle$ and $\left\langle\left(i^{\prime \prime}, k^{\prime \prime}\right),\left(j^{\prime \prime}, \ell^{\prime \prime}\right)\right\rangle$ both of which are associated to character $c$. Assume w.o.l.g. that $i^{\prime}>i^{\prime \prime}, k^{\prime}<k^{\prime \prime}, j^{\prime}<j^{\prime \prime}$ and $\ell^{\prime \prime}>\ell^{\prime}$. Then, there is a larger sub-rectangle $\left\langle\left(i^{\prime \prime}, k^{\prime}\right),\left(j^{\prime}, \ell^{\prime \prime}\right)\right\rangle$ of $R$ which contains both of the above rectangles, a contradiction. Hence, for any character $c$, a maximal sub-rectangle in $R$ is unique if it exists.

Lemma 1 permits us to define the following recursive algorithm for the MNDR problem:

We begin with the initial virtual rectangle $\langle(0,0),(n+1),(n+1)\rangle$. Suppose we are processing a rectangle $R$. For each character $c \in \Sigma$, we compute its maximal sub-rectangle $R_{c}$ in $R$ and recurse into $R_{c}$ until we meet one of the following conditions:

(1) There remains only a single point in $R_{c}$,

(2) There remains no point in $R_{c}$, or 
(3) $R_{c}$ is already processed.

The recursion depth clearly corresponds to the rectangle nesting depth, and we associate each $R$ with its maximum nesting depth $d_{R}$. Whenever we meet a rectangle $R_{c}$ with Condition (3), we do not recurse inside $R_{c}$ but simply return the already-computed maximum nesting depth $d_{R_{c}}$.

Initially, every rectangle $R$ is marked non-processed, and it gets marked processed as soon as the recursion for $R$ is finished and $R$ receives its maximum nesting depth. Each already processed rectangle remains marked processed until the end of the algorithm.

Theorem 2 Given two strings $A$ and $B$ of length $n$ over an integer alphabet of polynomial size in $n$, we can solve the MNDR problem (and hence the 2-LCPS problem) in $O\left(\sigma M^{2}+n\right)$ time and $O\left(M^{2}+n\right)$ space, where $\sigma$ denotes the number of distinct characters occurring in both $A$ and $B$.

Proof. To efficiently perform the above recursive algorithm, we conduct the following preprocessing (alphabet reduction) and construct the two following data structures.

Alphabet reduction: First, we reduce the alphabet size as follows. We radix sort the original characters in $A$ and $B$, and replace each original character by its rank in the sorted order. Since the original integer alphabet is of polynomial size in $n$, the radix sort can be implemented with $O(1)$ number of bucket sorts, taking $O(n)$ total time. This way, we can treat $A$ and $B$ as strings over an alphabet $[1,2 n]$. Further, we remove all characters that occur only in $A$ from $A$, and remove all characters that occur only in $B$ from $B$. Let $\hat{A}=\hat{a}_{1} \cdots \hat{a}_{\hat{m}}$ and $\hat{B}=\hat{b}_{1} \cdots \hat{b}_{\hat{n}}$ be the resulting strings, respectively. It is clear that we can compute $\hat{A}$ and $\hat{B}$ in $O(n)$ time. The key property of the shrunk strings $\hat{A}$ and $\hat{B}$ is that since all $M$ matching position pairs in the original strings $A$ and $B$ are essentially preserved in $\hat{A}$ and $\hat{B}$, it is enough to work on strings $\hat{A}$ and $\hat{B}$ to solve the original problem. If $\sigma$ is the number of distinct characters occurring in both $A$ and $B$, then $\hat{A}$ and $\hat{B}$ are strings over alphabet $[1, \sigma]$. It is clear that $\sigma \leq \min \{\hat{m}, \hat{n}\} \leq n$.

Data structure for finding next maximal sub-rectangles: For each character $c \in[1, \sigma]$, let $\mathcal{P}_{\hat{A}, c}$ and $\mathcal{P}_{\hat{B}, c}$ be the set of positions of $\hat{A}$ and $\hat{B}$ which match $c$, namely, $\mathcal{P}_{\hat{A}, c}=\left\{i \mid a_{i}=c, 1 \leq i \leq \hat{m}\right\}$ and $\mathcal{P}_{\hat{B}, c}=\left\{i \mid b_{i}=c, 1 \leq i \leq \hat{n}\right\}$. Then, given a rectangle $R$, finding the maximal sub-rectangle $R_{c}$ for character $c$ reduces to two predecessor and two successor queries on $\mathcal{P}_{\hat{A}, c}$ and $\mathcal{P}_{\hat{B}, c}$ We use two tables of size $\sigma \times \hat{m}$ each which answer predecessor/successor queries on $\hat{A}$ in $O(1)$ time. Similarly, we use two tables of size $\sigma \times \hat{n}$ each which answer predecessor/successor queries on $\hat{B}$ in $O(1)$ time. Such tables can easily be constructed in $O(\sigma(\hat{m}+\hat{n}))$ time and occupy $O(\sigma(\hat{m}+\hat{n}))$ space. Notice that for any position $i$ in $\hat{A}$ there exists a matching position pair $(i, k)$ for some position $k$ in $\hat{B}$, and vice versa. Therefore, we have $\max \{\hat{m}, \hat{n}\} \leq M$. Since $\sigma \leq \min \{\hat{m}, \hat{n}\} \leq \max \{\hat{m}, \hat{n}\}$, we have $\sigma(\hat{m}+\hat{n})=O\left(M^{2}\right)$. Hence the data structure occupies $O\left(M^{2}\right)$ space and can be constructed in $O\left(M^{2}\right)$ time.

Data structure for checking already processed rectangles: To construct a space-efficient data structure for checking if a given rectangle is al- 
ready processed or not, we here associate each character $\hat{A}$ and $\hat{B}$ with the following character counts: For any position $i$ in $\hat{A}$, let $\operatorname{cnt}_{\hat{A}}(i)=\mid\left\{i^{\prime} \mid \hat{a}_{i^{\prime}}=\right.$ $\left.\hat{a}_{i}, 1 \leq i^{\prime} \leq i\right\} \mid$ and for any position $k$ in $\hat{B}$, let $\operatorname{cnt}_{\hat{B}}(k)=\mid\left\{k^{\prime} \mid \hat{B}_{k^{\prime}}=\right.$ $\left.\hat{B}_{k}, 1 \leq k^{\prime} \leq k\right\} \mid$. For each character $c \in[1, \sigma]$, let $M_{c}$ denotes the number of matching position pairs between $\hat{A}$ and $\hat{B}^{\prime}$ for character $c$. We maintain the following table $T_{c}$ of size $M_{c} \times M_{c}$ : For any two matching positions pairs $(i, k)$ and $(j, \ell)$ for character $c$ (namely, $\hat{a}_{i}=\hat{b}_{k}=\hat{a}_{j}=\hat{b}_{\ell}=c$ ), we set $T_{c}\left[\operatorname{cnt}_{\hat{A}}(i), \operatorname{cnt}_{\hat{B}}(k), \operatorname{cnt}_{\hat{A}}(j), \operatorname{cnt}_{\hat{A}}(\ell)\right]=0$ if the corresponding rectangle $\langle(i, k),(j, \ell)\rangle$ is non-processed, and set $T_{c}\left[\operatorname{cnt}_{\hat{A}}(i), \operatorname{cnt}_{\hat{B}}(k), \operatorname{cnt}_{\hat{A}}(j), \operatorname{cnt}_{\hat{A}}(\ell)\right]=1$ if the corresponding rectangle is processed. Clearly, this table tells us whether a given rectangle is processed or not in $O(1)$ time. The total size for these tables is $\sum_{c \in[1, \sigma]} M_{c}^{2}=O\left(M^{2}\right)$.

We are now ready to show the complexity of our recursive algorithm.

Main routine: A unique visit to a non-processed rectangle can be charged to itself. On the other hand, each distinct visit to a processed rectangle $R$ can be charged to the corresponding rectangle which contains $R$ as one of its maximal sub-rectangles. Since we have $O\left(M^{2}\right)$ rectangles, the total number of visits of the first type is $O\left(M^{2}\right)$. Also, since we visit at most $\sigma$ maximal sub-rectangles for each of the $M^{2}$ rectangles, the total number of visits of the second type is $O\left(\sigma M^{2}\right)$. Using the two data structures described above, we can find each maximal sub-rectangle in $O(1)$ time and can check if it is already processed or not in $O(1)$ time. For each rectangle after recursion, it takes $O(\sigma)$ time to calculate the maximum nesting depth from all of its maximal sub-rectangles. Thus, the main routine of our algorithm takes a total of $O\left(\sigma M^{2}\right)$ time.

Overall, our algorithm takes $O\left(\sigma M^{2}+n\right)$ time and uses $O\left(M^{2}+n\right)$ space.

\section{Conclusions and further work}

In this paper, we studied the problem of finding a longest common palindromic subsequence of two given strings, which is called the 2-LCPS problem. We proposed a new algorithm which solves the 2-LCPS problem in $O\left(\sigma M^{2}+n\right)$ time and $O\left(M^{2}+n\right)$ space, where $n$ denotes the length of two given strings $A$ and $B, M$ denotes the number of matching position pairs of $A$ and $B$, and $\sigma$ denotes the number of distinct characters occurring in both $A$ and $B$.

Since the 2-LCPS problem is at least as hard as the well-studied 4-LCS problem, and since any known solution to the 4-LCS problem takes at least $O\left(n^{4}\right)$ time in the worst case, it seems a big challenge to solve the 2-LCPS problem in $O\left(M^{2-\lambda}\right)$ or $O\left(n^{4-\lambda}\right)$ time for any constant $\lambda>0$. This view is supported by the recent result on a conditional lowerbound for the $k$-LCS problem: If there exists a constant $\lambda>0$ and an integer $k \geq 2$ such that the $k$-LCS problem over an alphabet of size $O(k)$ can be solved in $O\left(n^{k-\lambda}\right)$ time, then the famous SETH (strong exponential time hypothesis) fails [6].

As an open problem, we are interested in whether the space requirement of our algorithms can be reduced, as this could be of practical importance. 


\section{References}

[1] D. Maier, The complexity of some problems on subsequences and supersequences, J. ACM 25 (2) (1978) 322-336.

[2] R. A. Wagner, M. J. Fischer, The string-to-string correction problem, J. ACM 21 (1) (1974) 168-173.

[3] V. Arlazarov, E. Dinic, M. Kronrod, I. Faradzev, On economical construction of the transitive closure of a directed graph, Soviet Math. Dokl. 11 (1970) 1209-1210.

[4] W. J. Masek, M. Paterson, A faster algorithm computing string edit distances, J. Comput. Syst. Sci. 20 (1) (1980) 18-31.

[5] S. Grabowski, New tabulation and sparse dynamic programming based techniques for sequence similarity problems, Discrete Applied Mathematics 212 (2016) 96-103.

[6] A. Abboud, A. Backurs, V. V. Williams, Tight hardness results for LCS and other sequence similarity measures, in: FOCS 2015, 2015, pp. 59-78.

[7] F. Y. L. Chin, A. D. Santis, A. L. Ferrara, N. L. Ho, S. K. Kim, A simple algorithm for the constrained sequence problems, Inf. Process. Lett. 90 (4) (2004) 175-179.

[8] A. N. Arslan, Regular expression constrained sequence alignment, J. Discrete Algorithms 5 (4) (2007) 647-661.

[9] C. S. Iliopoulos, M. S. Rahman, New efficient algorithms for the LCS and constrained LCS problems, Inf. Process. Lett. 106 (1) (2008) 13-18.

[10] G. Kucherov, T. Pinhas, M. Ziv-Ukelson, Regular language constrained sequence alignment revisited, Journal of Computational Biology 18 (5) (2011) 771-781.

[11] S. Deorowicz, Quadratic-time algorithm for a string constrained LCS problem, Inf. Process. Lett. 112 (11) (2012) 423-426.

[12] E. Farhana, M. S. Rahman, Doubly-constrained LCS and hybridconstrained LCS problems revisited, Inf. Process. Lett. 112 (13) (2012) $562-565$.

[13] D. Zhu, X. Wang, A simple algorithm for solving for the generalized longest common subsequence (LCS) problem with a substring exclusion constraint, Algorithms 6 (3) (2013) 485-493.

[14] E. Farhana, M. S. Rahman, Constrained sequence analysis algorithms in computational biology, Inf. Sci. 295 (2015) 247-257.

[15] D. Zhu, Y. Wu, X. Wang, An efficient algorithm for a new constrained LCS problem, in: ACIIDS 2016, 2016, pp. 261-267. 
[16] D. Zhu, Y. Wu, X. Wang, An efficient dynamic programming algorithm for STR-IC-STR-EC-LCS problem, in: GPC 2016, 2016, pp. 3-17.

[17] S. R. Chowdhury, M. M. Hasan, S. Iqbal, M. S. Rahman, Computing a longest common palindromic subsequence, Fundam. Inform. 129 (4) (2014) 329-340.

[18] S. Y. Itoga, The string merging problem, BIT 21 (1) (1981) 20-30.

[19] W. J. Hsu, M. W. Du, Computing a longest common subsequence for a set of strings, BIT 24 (1) (1984) 45-59.

[20] R. W. Irving, C. Fraser, Two algorithms for the longest common subsequence of three (or more) strings, in: CPM 1992, 1992, pp. 214-229.

[21] K. Hakata, H. Imai, The longest common subsequence problem for small alphabet size between many strings, in: ISAAC 1992, 1992, pp. 469-478.

[22] Q. Wang, D. Korkin, Y. Shang, A fast multiple longest common subsequence (MLCS) algorithm, IEEE Trans. Knowl. Data Eng. 23 (3) (2011) $321-334$. 